\title{
Modelling
}

\section{Serial lung model for simulation and parameter estimation in body plethysmography}

\author{
A. F. M. Verbraak' J. M. Bogaard ' J. E. W. Beneken? \\ E. Hoorn' A. Versprille'
}

Department of Pulmonary Biseases, University Hospital Dilkzigi: Rotierdam, The Netherlands 20ivision of Nedical Electrical Engineering, Enchoven University of Technology, The Netherlands

\begin{abstract}
A serial lung model with a compressible segment has been implemented to simulate different types of lung and airway disorders such as asthma, emphysema, fibrosis and upper airway obstruction. The model described can be used during normal breathing, and moreover the compliant segment is structured according to more recent phvsiological data. A parameter estimation technique was applied and its reliability and uniqueness were tested by means of sine wave input signals. The characteristics of the alveolar pressure/flow patterns simulated with the model agree to a great extent with those found in the literature. In the case of absence of noise the parameter estimation routine produced unique solutions for different simulated pathologic classes. The sensitivity of the different parameters depended on the values belonging to each class of pathology. Some more simplified models are presented and their advantages over the complex model in special types of pathology are demonstrated. Noise added to the simulated flow appeared to have no influence on the estimated parameters, in contradiction to the effects with noise added to the pressure signal. In that case effective resistance was accurately estimated. Where parameters had no influence, as for instance upper airway resistance in emphysema or peripheral airway resistance in upper airway obstruction, the measurement accuracy was less. In all other cases, a satisfactory accuracy could be obtained.
\end{abstract}

Keywords-Body plethysmography, Parameter estimation, Serial lung model, Simulation

Mod: \& Bial Eng \& Comput. 1991, 29, 309-317

\section{List of symbols}

$C$ compliance of the lung, litre $\mathrm{kPa}^{-1}$

$E E \quad$ square error

$K_{1} \quad$ linear resistance of the large airways, $\mathrm{kPa}_{\text {litre }}{ }^{-1} \mathrm{~s}$

$K_{2}$ turbulent resistance of the large airways, $\mathrm{kPa}$ litre $\mathrm{e}^{-2} \mathrm{~s}^{2}$

$K_{3} \quad$ linear resistance parameter model $2, \mathrm{kPa}$ litre $^{-1} \mathrm{~s}$

$K_{4}, K_{5}$ linear resistance parameters model 3 for inspiration and expiration, respectively, $\mathrm{kPa}$ litre $^{-1} \mathrm{~s}$

$K_{6}, K_{7}$ linear resistance parameters model 4 for inspiration and expiration, respectively, $\mathrm{kPa}$ litre ${ }^{-1} \mathrm{~s}$

$K_{8}, K_{9}$ turbulent resistance parameters model 4 for inspiration and expiration, respectively, $\mathrm{kPa}$ litre $^{-1} \mathrm{~s}^{2}$

$R_{e f f} \quad$ effective resistance, $\mathrm{kPa}$ litre ${ }^{-1} \mathrm{~s}$

$R_{c} \quad$ linear resistance of the compressible segment, $\mathrm{kPa}$ litre $^{-1} \mathrm{~s}$

$R_{\mathrm{c} 0} \quad$ weighting constant (see text), $\mathrm{kPa}$ litre ${ }^{-1} \mathrm{~s}$

$R_{p} \quad$ resistance line trough extreme pressure points, $\mathrm{kPa}$ litre $^{-1} \mathrm{~s}$

$R_{s} \quad$ linear resistance of the small airways, $\mathrm{kPa}$ litre $\mathrm{C}^{-1} \mathrm{~s}$

First received 1st June 1988 and in final form 9th April 1990

(C) IFMBE: 1991
$R_{u} \quad$ total resistance of the upper airways, $\mathrm{kPa}$ litre ${ }^{-1} \mathrm{~s}$

$P_{A} \quad$ alveolar pressure, $\mathrm{kPa}$

$P_{L} \quad$ elastic lung recoil, $\mathrm{kPa}$

$P_{L, F R C} \quad$ elastic lung recoil at FRC, $\mathrm{kPa}$

$P_{t m} \quad$ transmural pressure, $\mathrm{kPa}$

$P_{p l} \quad$ pleural pressure, $\mathrm{kPa}$

$P_{s} \quad$ pressure drop over the small airways, $\mathrm{kPa}$

$V$ alveolar volume, litre

$V_{L} \quad$ alveolar volume + volume of the airways, litre

$V_{c} \quad$ volume of the compressible segment, litre

$V_{c N} \quad$ maximum volume of the compressible segment for normals, litre

$V_{A}^{\prime} \quad$ flow into the alveolar space, litre $\mathrm{s}^{-1}$

$V_{m}^{\prime} \quad$ flow at the mouth, litre $\mathrm{s}^{-1}$

$V_{c 0}^{m} \quad$ weighting constant (see text), litre

$P_{t m s} \quad$ point of curvature for the compressible segment, $\mathrm{kPa}$

\section{Introduction}

A DIRECT calculation of airway resistance from driving pressure and airflow was enabled by body plethysmog- 
raphy. Because the difference between the volume displacement at the mouth and the simultaneous change in chest volume are caused by compression or expansion of intrathoracic gas, the plethysmograph can be used for measurements of thoracic gas volume and the alveolar pressure $P_{A}$ inside the thorax. In 1956, DuBoIs et al. re-established the method for routine clinical use.

By plotting $P_{A}$ against simultaneously measured flow at the mouth $V_{m}^{\prime}$, a pressure/flow loop was obtained. Only when $P_{A}$ and $V_{m}^{\prime}$ yield a linear relationship can airway resistance $R$ be derived directly according to Ohm's law $R=P_{A} / V_{m}^{\prime}$, where ambient pressure is reset to zero. However, for different types of pulmonary pathology, nonlinearities were found between $P_{A}$ and $V_{m}^{\prime}$, not permitting such a direct derivation of $R$.

In 1971, MATTHYS made a classification of patterns that were caused by different distributions of elastic and resistive properties of the lung, and by the pressure and flow differences in different parts of the lung.

Usually, estimates of airway resistance are obtained graphically from the body plethysmographic pressure/flow diagrams. REINERT et al. (1975) restricted their analysis to the linear parts of pressure/flow curves. Others have estimated airway resistance from a few discrete data, as e.g. total resistance $R_{t}$ which is calculated from the line through the two extreme values of pressure in the inspiratory and expiratory part of the curve (ULMER and REIF, 1965 ) and from lines through mean pressure points at flow rates of 0.5 litre $\mathrm{s}^{-1}$ during inspiration and expiration (GuYatT et al., 1967). In 1975, SMIDT et al. proposed to calculate an effective resistance variable, $R_{\text {eff }}$, which was related to the dissipation of energy during the whole respiratory cycle. $R_{e f f}$ was defined as the integral of pressure times volume increments divided by the integral of flow times volume increments. This variable is comparable with the effective resistance as defined in alternating current theory. Holland et al. (1986) showed that $R_{e f f}$ was almost independent of those errors in $P_{A}$ which were associated with differences in humidity and temperature between inspired and expired air.

System identification and parameter estimation on input/output data receive increasing acceptance in physiological research (BEKEY and BENEKEN, 1978). Until now only a few papers in the field of body plethysmography have dealt with modelling techniques to estimate the physiological variables describing lung mechanics. BANERJEE et al. (1976) and CETTL et al. (1979) attributed the degree of looping of the $P_{A}-V_{m}^{\prime}$ patterns to parallel inhomogeneity and modelled it by two $R C$ branches in parallel, as described by OTIS et al. (1956). Another group of authors (PrIDE et al., 1967, FeINBERG et al., 1970; FEINBERG and CHESTER, 1972; GoldEN et al., 1973) modelled a serial inhomogeneity, describing the lung as an alveolar compartment, lower and upper airways and a compliant segment in between, allowing a simulation of airway compression during expiration. The serial model of FEINBERG et al. (1970) was based on a comprehensive mathematical basis to obtain a quantitative indication of various aspects of lung mechanics. An estimation routine fitted the model to the data obtained during panting. GoLDEN et al. (1973) used the same type of model with a restricted number of parameters.

The serial models published so far are dependent on panting manoeuvres to approximate a constant lung volume during the measurements. We have extended the lung model described by GoLDEN et al. (1973), with lung compliance dependent on lung volume, to adapt it to the circumstances of normal breathing. Additionally, we have improved the model by giving a better physiological basis for the compressible segment based on more recent publications. Furthermore, we have evaluated the sensitivity and the uniqueness of the estimated parameters and the influence of noise.

\section{Theory and methods}

\subsection{The serial lung model}

For the definition of the model the functional approach of GoldEN et al. (1973) was used. The model (Fig. 1) was restricted to serial inhomogeneity with respect to both resistance and elastic properties of the airways. All alveoli were lumped to one compartment $V_{A}$, which was connected to ambient air by a tube representing the lumped airways. This tube consisted of three parts in series: one for the small airways, a compressible segment in between, and one for the large airways. The alveolar compartment and the intrapulmonary airways were influenced by the intrathoracic pressure, which was equal to pleural pressure $P_{p l}$. Golden et al. (1973) assumed static lung recoil $P_{L}$ to be constant because of the small lung volume changes

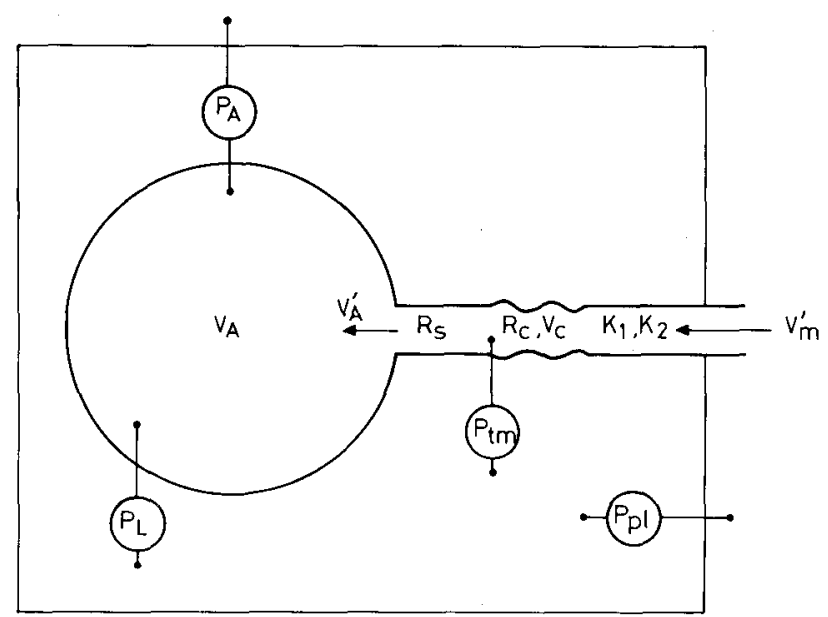

Fig. 1 The serial lung model. For the explanation of the different variables and symbols see list of symbols and text

during the panting manoeuvres. The elastic properties were modelled such that the static lung recoil $P_{L}$ increased when alveolar volume $V_{A}$ increased. In the range of normal breathing it was assumed that the increase of $P_{L}$ against the change in alveolar volume yields:

$$
P_{L}(t)=P_{L, F R C}(0)+1 / C \int_{\sigma=0}^{t} V_{A}^{\prime}(\sigma) d \sigma
$$

where $C$

$$
P_{L, F R C}(0) \text { is the } P_{L}(\mathrm{kPa}) \text { at functional residual }
$$
capacity (FRC), which is the lung volume at the end of normal expiration, and thus at the beginning of normal inspiration

$t=0 \quad$ is the moment at which inspiration starts.

$V_{A}^{\prime}(\sigma) \quad$ is flow in litre $\mathrm{s}^{-1}$ into the alveolar compartment at time $t=\sigma$, giving inspiratory volume $V_{I}$ at any value of $t$ by integration.

In the case of normal breathing, as in our model, the magnitude of the flow is such that the pressure drop produced by convective acceleration can be neglected, and so airway pressure at different sites in the airways is assumed to be a function of frictional pressure loss only. 


\subsection{Airway resistances}

The resistance of the upper airways $R_{u}$ was modelled as a rigid part. $R_{u}$ included a flow-dependent term, and was expressed by the 'Rohrer equation' (ROHRER, 1915):

$$
P_{u}=R_{u} V_{m}^{\prime}=K_{1} V_{m}^{\prime}+K_{2} V_{m}^{\prime 2}
$$

where $P_{u} \quad$ is pressure drop in the upper airways

$K_{1}$ is a factor related to the pressure drop due to laminar flow in the upper airways, in $\mathrm{kPa}$ litre ${ }^{-1} \mathrm{~s}$

$K_{2}$ is a factor related to the pressure drop due to turbulent flow in the upper airways, in $\mathrm{kPa}$ litre ${ }^{-2} \mathrm{~s}^{2}$

$V_{m}^{\prime}$ is the flow at the mouth, in litre $\mathrm{s}^{-1}$.

The resistance of the peripheral airways $R_{s}$ was assumed to be constant and we neglected the influence of the lung volume changes during the breathing manoeuvre at volumes above FRC. Based on the dimensions of these airways a Poiseuille flow was assumed. So, the linear pressure drop $P_{s}$ in these airways is given by

$$
P_{s}=R_{s} V_{A}^{\prime}
$$

where $R_{s}$ is the resistance of the small airways, kPa litre ${ }^{-1} \mathrm{~s}$.

The resistance of the compressible segment represented the resistance in those airway generations, which are susceptible to the changes in transmural pressure $P_{t m}$ being the difference between intrabronchial and pleural pressure. The functional behaviour of the compressible segment was modelled as a nonlinear relationship between $P_{t m}$ and its cross-sectional area. $P_{t m}$ of the compressible segment was located between this segment and the peripheral airways and was assumed to be the same over the whole segment. Furthermore, a constant length and an equal crosssectional area over its length is assumed. Thus, the crosssectional area can be expressed in terms of volume $V_{c}$. Assuming a laminar flow the resistance in a tube of constant length will be proportional to $1 / r^{4}$ and thus to $1 / V_{c}^{2}$. $V_{c}$ was normalised to the maximum volume $V_{c N}$ of the compressible segment for the normal lung, having a recoil tension of $0.5 \mathrm{kPa}$ at FRC-level.

The largest changes in volume will occur for changes in $P_{t m}$ around zero. The site in the segment where $P_{t m}$ equals zero is called the equal pressure point (EPP) (MEAD et al., 1967; HyATT, 1983). The location of the EPP depends on the elasticity of the lung and the peripheral airway resistance $R_{s}$. This dependence is described by eqns. (4-7).

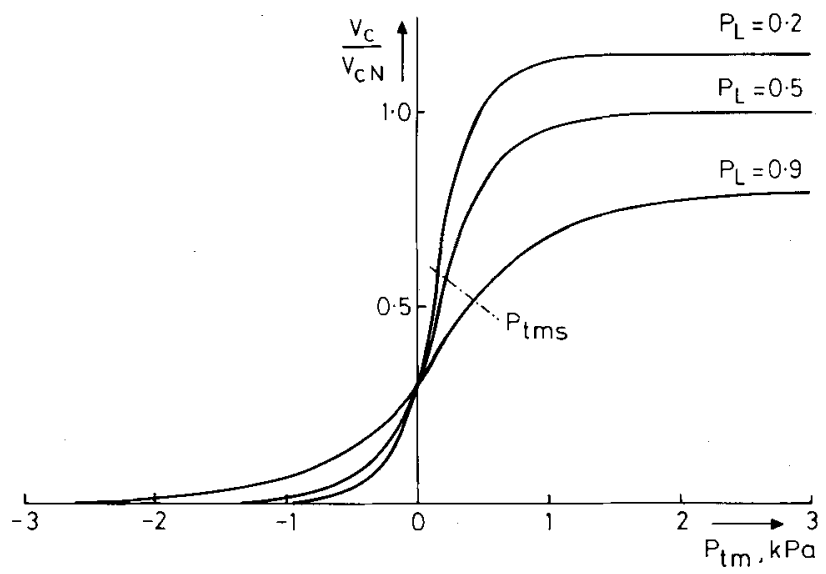

Fig. 2 The relationship between the normalised volume of the compressible segment $\left(V_{c} / V_{c N}\right)$ and the transmural pressure $P_{t m}$ with the elastic lung recoil as parameter. For symbols see list of symbols and text

$$
P_{t m}(x)=P_{b r}(x)-P_{p l}
$$

where $P_{b r}(x)$ is intrabronchial pressure (kPa) as a function of the location $x$ in the airways

$P_{p l} \quad$ is pleural pressure in $\mathrm{kPa}$.

If during breathing eqn. 3 is valid then

$$
P_{b r}(x)=P_{A}+R_{s}(x) V_{A}^{\prime}
$$

where $R s(x)$ is the cumulated airway resistance in $\mathrm{kPa}$ litre ${ }^{-1} \mathrm{~s}$ from the alveolus to the location $x$ in the airways

$P_{p l} \quad$ is the sum of elastic recoil $P_{L}$ and alveolar $P_{A}$

$$
P_{p l}=P_{L}+P_{A}
$$

From eqns. 4, 5 and 6

$$
P_{t m}(x)=P_{L}+R_{s}(x) V_{A}^{\prime}
$$

EPP will be found at the location $x$ where $P_{t m}(x)=0$. Then the driving pressure for the flow is given by $P_{L}$ and is determined by the peripheral airway resistance $R_{s}(x)$. This means that the EPP is shifted upstream or downstream, dependent on elastic recoil and/or peripheral airway resistance.

The stiffiness of airways increases from the alveoli towards the mouth (LAMBERT et al., 1982). The model described neither the location in detail of the EPP within the tapered serial system with gradually changing $\boldsymbol{R}_{s}$, nor the dependence of the airway compliance on generation number. Therefore, it is necessary to find another solution for the modelling of the changing properties of the compressible segment in dependence on the location $x$ of the EPP. A low $P_{L}$ means a more peripheral located and less stiff compressible segment with a larger volume at maximum distension. Therefore, we modelled the elastic properties of the compressible segment in dependence on the $P_{L}$ values.

The mathematical description of the volume behaviour of the compressible segment in terms of $P_{t m}$ (Fig. 2) is as follows:

$$
\begin{aligned}
P_{t m} & \geqslant P_{t m s} \quad \frac{V_{c}}{V_{c N}}=S-\frac{S^{2}}{1.2} \times\left[\frac{0.6}{S}\right]^{\left(P_{t m} / P_{t m s}\right)} \\
P_{t m} & <P_{t m s} \quad \frac{V_{c}}{V_{c N}}=0.3 \times\left[\frac{0.6}{S}\right]^{\left(-P_{\left.t m / P_{t m s}\right)}\right.} \\
P_{t m s} & =0.15+0.05 P_{L} \\
S & =1.25-P_{L} / 2
\end{aligned}
$$

where $S$ is an auxiliary variable to optimise calculations

Eqns. $(8 a-9 b)$ describe the relationship between the transmural pressure over the compressible segment and the volume of this segment, with elastic lung recoil as a parameter. These relationships are adapted from the literature and have been based on measurements in dogs and extrapolated to humans (MARTIN and PROCTOR, 1958; HyatT and Flath, 1966; MARTin et al., 1965; MACKLEM and MEAD, 1968; MurTaGH et al., 1971). For mathematical convenience the curves were considered to be sigmoidal and split up into an upper and a lower part with an intersection at $P_{t m}=P_{t m s}$, where $P_{t m s}$ is the point of curvature. $P_{t m s}$ depends on elastic lung recoil (eqn. $9 a$ ). A continuous set of curves was used over the whole range of static lung recoil values. The volume of the compressible segment $V_{c 0}$ at maximum transmural pressure for $P_{L}=0.5 \mathrm{kPa}$ was chosen as 0.125 litre and the resistance of the compressible airways $R_{c 0}$ was chosen as $0.06 \mathrm{kPa}$ litre ${ }^{-1} \mathrm{~s}$, as an estimate for the airway generations 2-10 (LAMBERT et al., 
1982). The choice of these generations within the 23 generations of the bronchial tree is also based on the experimental evidence for the location of the EPP. In Fig. 2 the normalised values of $V_{C}$ are plotted against $P_{t m}$ for three values of $P_{L}(0.2,0.5$ and 0.9$)$.

The flow at the mouth is dependent on the peripheral airway resistance $R_{s}$, the resistance of the compressible segment $R_{c}$ the upper airway resistance $R_{u}$ and alveolar, pleural and elastic recoil pressures $P_{A}, P_{p l}$ and $P_{L}$. For the complete mathematical description of the lung model with a compressible segment, lung model 1 is represented by the following equations, which can be derived:

Model 1:

$$
\begin{aligned}
& P_{p l}=P_{A}-P_{L} \\
& P_{L}-P_{t m}+R_{S} V_{A}^{\prime}=0 \\
& P_{t m}+P_{p l}+\left(R_{c}+K_{1}\right) V_{m}^{\prime}+K_{2}\left(V_{m}^{\prime}\right)^{2}=0 \\
& R_{c}=R_{c 0}\left(V_{c 0} / V_{c}\right)^{2}
\end{aligned}
$$

where $R_{c}$ is the resistance of the compressible segment in $\mathrm{kPa}$ litre ${ }^{-1} \mathrm{~s}$. $V_{\mathrm{c}}$ is the volume of the compressible segment in litres. $R_{c 0}$ and $V_{c 0}$ are model constants as defined before. $R_{c 0}$ defines the overall magnitude of $R_{c}$, and $V_{c 0}$ determines the relative volume of the compressible segment at which $R_{c}$ will be of influence.

$$
\begin{aligned}
& V_{c}(t)=V_{c}(0)+\int_{\sigma=0}^{t}\left(V_{m}^{\prime}-V_{A}^{\prime}\right) d \sigma \\
& P_{L}(t)=P_{L, F R C}+1 / C \int_{\sigma=0}^{t} V_{m}^{\prime} d \sigma \\
& P_{t m}=f\left(V_{c}, P_{L}\right)
\end{aligned}
$$

where $V_{A}^{\prime}$ is the flow into the alveolar compartment litre $s^{-1}$

$V_{m}^{\prime}$ is the flow at the mouth, litre $\mathrm{s}^{-1}$

$R_{s}, P_{L}, K_{1}$ and $K_{2}$ are the model parameters.

\subsection{Simplified models}

In cases where the influence of compressibility of the airways could be neglected, e.g. stiff lungs and normal lungs at low flow, simpler models were used with a restricted number of parameters if compared with the basic model. Models 2, 3 and 4 were defined by an absence of compressibility and different dominant properties determining the behaviour of model 1 .

Model 2: a linear resistance parameter $K_{3}\left(\mathrm{kPa}\right.$ litre $\left.{ }^{-1} \mathrm{~s}\right)$ for inspiration and expiration is introduced:

$$
P_{A}=K_{3} V_{m}^{\prime}
$$

Model 3: different linear resistances parameters $K_{4}$ and $K_{5}\left(\mathrm{kPa}\right.$ litre $\left.^{-1} \mathrm{~s}\right)$ for inspiration and expiration are introduced:

$$
\begin{aligned}
& P_{A, \text { in }}=K_{4} V_{m, \text { in }}^{\prime} \\
& P_{A, \text { ex }}=K_{5} V_{m, \text { ex }}^{\prime}
\end{aligned}
$$

where $P_{A, \text { in }}$ is alveolar pressure during inspiration in $\mathrm{kPa}$

$$
\begin{array}{ll}
P_{A, e x} & \text { is alveolar pressure during expiration in } \\
\mathrm{kPa}
\end{array}
$$

Model 4: different linear and turbulent resistance parameters $\left(K_{6}\right.$ and $K_{7}, \quad \mathrm{kPa}$ litre ${ }^{-1} \mathrm{~s}$, and $K_{8}, K_{9}$, $\mathrm{kPa}$ litre $\mathrm{s}^{2}$ ) are used for inspiration and expiration, respectively:

$$
P_{A, \text { in }}=K_{6} V_{m, \text { in }}^{\prime}+K_{8} V_{m, \text { in }}^{\prime 2}
$$

$$
P_{A, e x}=K_{7} V_{m, e x}^{\prime}+K_{9} V_{m, e x}^{\prime 2}
$$

\subsection{Parameter estimation}

The parameter estimation technique and the simulation of the serial lung model were connected in the way indicated in Fig. 3. With the serial lung model (simulation) an output signal $V_{m}^{\prime}$ was calculated for a given $P_{A}$. Based on the same stimulus $P_{A}$ and with use of one of the described mathematical models $(1,2,3$ or 4$)$ the computer calculated (estimation) a predicted response $V_{\text {pred }}^{\prime}$ for a given set of start parameters. Then, a new set of parameters was computed to reduce the difference in square error $E E$ between $V_{p r e d}^{\prime}$ and the known $V_{m}^{\prime}$. This was continued either for a maximum predefined number of 20 iterations or for a smaller number when the difference in square error $E E$ between the two responses was reduced below 0.0001 .

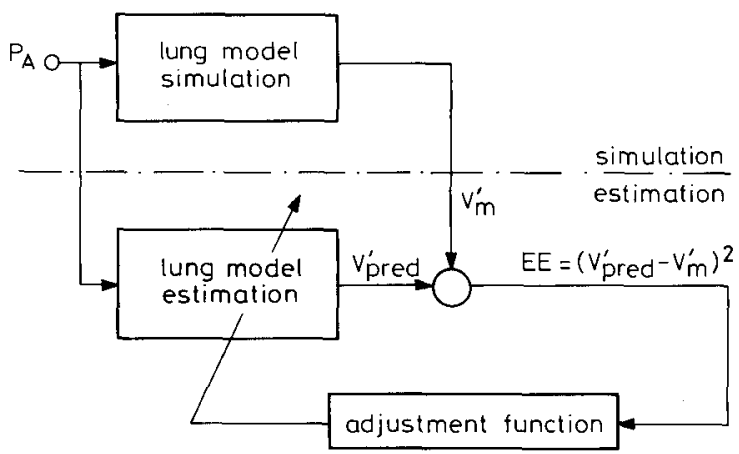

Fig. 3 The parameter estimation process; for explanation see text

For the parameter estimation technique the Marquardt algorithm (MARQUARDT, 1963) was applied, which is a modified Gauss-Newton algorithm. The loss function $E E=\left(V_{\text {pred }}^{\prime}-V_{m}^{\prime}\right)^{2}$ was minimised. With the Marquardt algorithm the step size of each adjustment can be influenced by means of a factor $\mu$. By changing $\mu$ after each iteration it was possible to control dynamically the convergence of the estimation $(\mu=0$, Gauss Newton; $\mu=\infty$ steepest descent with step size zero). At the start of the estimation $\mu$ was set at $0 \cdot 1$. If the error was smaller in the next iteration, $\mu$ was adjusted to $0.4 \mu$, and if the error was larger the last iteration was repeated after $\mu$ was changed to $10 \mu$. The factors 0.4 and 10 were found empirically.

\subsection{Forcing the serial model with simulated signals}

First the serial model was stimulated by a sinusoidally changing $P_{A}$. Because of unequal inspiratory and expiratory resistances (models 1, 3 and 4) the sinusoidal alveolar pressure curve had to be corrected to minimise the difference $(\Delta V)$ between the lung volume at the start $(\sigma=0)$ and that at the end $(\sigma=t)$ of the simulated respiratory cycle below 0.01 litre. The change in lung volume $\Delta V$ between the start and end of the respiratory cycle was found from the value obtained after integration of the output $V_{m}^{\prime}$ over the whole respiratory cycle. The minimisation of $\Delta V$ was realised by the introduction of an offset in the zero line of the sinusoidal alveolar pressure signal.

A higher expiratory than inspiratory resistance needed a shift of the zero line into the direction of the inspiratory pressure curve, giving a larger amplitude and duration of the expiratory pressure curve. For the inspiratory part of the curve the opposite changes occurred. The offset in the zero line was calculated, from the volume shift $\Delta V$ and the overall resistance $R_{\text {eff }}$ (HollaND et al., 1986), by an iterative process. 


\subsection{Choice of parameters for testing the simulation}

The parameter values for model 1 were divided into five classes: the normal lung $(N)$, the emphysematous lung $(E)$, the fibrotic lung $(F)$, the asthmatic lung $(A)$ and the lung with a large increase in upper airway resistance $(U)$. The characteristic values for the various parameters were chosen from reviews of pulmonary pathophysiology (PRIDE et al., 1967; PRIDE 1971; CoTEs, 1975; OTIS et al., 1956, and those for upper airway resistance for class $U$ were derived from patients with bilateral vocal cord paralysis (BOGAARD et al. 1987a; b).

Emphysema was modelled by a lower value of the elastic recoil $P_{L, F R C}$ and thus an increased lung compliance $(C)$. Because of a lower $P_{L, F R C}$ the traction on the walls of the respiratory bronchioles is smaller and consequently pleural pressure will be less negative with respect to alveolar pressure (eqn. 6), causing a narrowing of the small airways and therefore an increase in $R_{s}$. In fibrosis the characteristic physiological disorder is the increased stiffness of the lung tissue, which was modelled by an increase of $P_{L, F R C}$ and a decrease of $C$. In asthma both upper and peripheral airway resistances are increased, which was reflected in increased values for $K_{1}, K_{2}$ and $R_{s}$. To simulate patients with an upper airway obstruction only $K_{1}$ and $K_{2}$ were greatly increased, according to the results obtained from patients with bilateral vocal-cord paralysis as mentioned above.

\subsection{Uniqueness}

The validity of the parameters obtained by a parameter estimation technique depends on the uniqueness of the parameter values. To evaluate the uniqueness, simulations were made for the five classes $N, E, F, A$ and $U$ as mentioned in Table 1. The uniqueness of the calculated parameter set and the convergence of the iterative estimation procedure were analysed by estimating the parameters of a class when starting from the parameter values belonging to the other classes, e.g. simulation of $N$, and starting of parameters $E, F, A$ and $U$, respectively.

Table 1 Values for the parameters $\left(K_{1}, K_{2}, R_{s}\right.$ and $\left.P_{L}\right)$ and lung compliance $C$ for the different groups $N, E, F, A$ and $U$

\begin{tabular}{cccccc}
\hline & $\begin{array}{c}K_{1}, \\
\mathrm{kPalitre}^{-1} \mathrm{~s}\end{array}$ & $\begin{array}{c}K_{2}, \\
\mathrm{kPa} \mathrm{litre}^{-2} \mathbf{s}^{2}\end{array}$ & $\begin{array}{c}R_{s} \\
\mathrm{kPa} \mathrm{litre}^{-1} \mathrm{~s}\end{array}$ & $\begin{array}{c}P_{\mathrm{L}, F R C}, \\
\mathbf{k P a}\end{array}$ & $\begin{array}{c}C, \\
\text { litre kPa }\end{array}$ \\
\hline$N$ & 0.05 & 0.02 & 0.03 & 0.5 & 2.0 \\
$E$ & 0.05 & 0.02 & 0.3 & 0.1 & 5.0 \\
$F$ & 0.05 & 0.02 & 0.03 & 0.9 & 1.0 \\
$A$ & 0.1 & 0.06 & 0.3 & 0.5 & 2.0 \\
$U$ & 0.29 & 4.6 & 0.03 & 0.5 & 2.0 \\
\hline
\end{tabular}

\subsection{Sensitivity}

The squared error $E E=\left(V_{\text {pred }}^{\prime}-V_{m}^{\prime}\right)^{2}$ was determined after application of stepwise changes in the parameters in order to study the sensitivity of the parameter estimation technique. This was done in two ways:

(i) by determination of the interdependence of pairs of parameters

(ii) by studying the influence of fluctuations in each of the parameters on the resulting squared error.

\subsection{Noise}

Random noise was applied to the input signal of alveolar pressure and to the output signal of flow of the serial lung model. The mean noise level was $0.02 \mathrm{kPa}$ for the pressure and 0.02 litre $\mathrm{s}^{-1}$ for the flow. The deviation of the parameter estimates in the different classes from the selected input parameter values with which the simulation was performed was investigated by starting with values of the class itself and values obtained from the normal situation. For the normal situation the starting values for the emphysema class were also applied. The noise was generated by a noise generator of Digital Equipment Corporation which was implemented in the Fortran-IV library belonging to the RT-11 operating system.

\section{Results}

The results of simulations of the different pathological classes as defined in Table 1 are presented in Fig. 4. These simulations were based on a sinusoidal alveolar pressure change with an amplitude of $0.5 \mathrm{kPa}$ for classes $N, E, A$ and $U$ and $0.2 \mathrm{kPa}$ for class $F$, all at a rate of $0.5 \mathrm{~Hz}$. The lower values of alveolar pressure in class $F$ are necessary for the calculation of the segment volume with the Runge Kutta algorithm in the case of an almost stiff compressible segment because of high $P_{L}$ values. The difference between the maximum of alveolar pressure in inspiration and that in expiration for emphysema (Fig. $4 E$ ) was caused by a shift of the zeroline in the alveolar pressure. This shift was necessary to obtain an equal inspiratory and expiratory volume, as mentioned before. The characteristics of the simulated curves were in agreement with recordings of normals and patients, as documented among others by MATTHYs et al.(1971; 1979).

For each curve the resistance $R_{t}$ and the effective resistances for inspiration $\left(R_{e f f, i}\right)$, expiration $\left(R_{e f f, e}\right)$ and total ventilatory cycle $\left(R_{\text {eff }}\right)$ were calculated. To illustrate the mechanical behaviour of a lung with emphysema, the input variable $P_{A}$, the output variable $V_{m}^{\prime}$ and the resulting variables $P_{t m}$ and $R_{c}$ were plotted as a function of time for both classes $E$ and $N$ (Fig. 5). The offset in the zero line of alveolar pressure (Fig. 5a) compensated for a higher overall expiratory resistance (Fig. $5 d$ ) leading to a smaller peak flow $V_{m}^{\prime}$ (Fig. $5 b$ ). In case of emphysema $P_{t m}$ was around zero during a part of the expiratory phase (Fig. $5 c$ ). In this part the calculated resistance $\left(R_{c}\right)$ of the collapsible segment was relatively large (Fig. $5 d$ ) For the fibrotic case, not shown, the resistance $R_{c}$ was almost constant during the respiratory cycle.

In the estimation procedure the parameters used in the simulations could be determined, independent of the start parameters.

For classes $E$ and $F$ the dependence between the parameters is shown in Fig. 6. In this figure the contour lines where parameter combinations have the same loss function $E E$, were plotted. In each plot the parameters $K_{1}$ and $R_{s}$ fluctuated around their optimal values of 0.05 and 0.3 , respectively. The parameter $P_{L}$ had different values, 0.1 and 0.9 , respectively. The parameter $K_{2}$ was 0.02 and was constant in both cases. In the simulation of fibrosis the contour lines were almost straight, whereas in emphysema they were more elliptical. This implied that for fibrosis $K_{1}$ and $R_{s}$ were almost completely interdependent, i.e. cannot be estimated separately.

Fig. 7 presents the $E E$ changes for the $N$ and $F$ class, after individually changing the four parameters between 50 per cent and 150 per cent of their optimal values for each class. From this figure it can be noticed that the influence of a change in the parameter values $K_{1}, K_{2}$ and $R_{s}$ on the squared error was different for the two classes $N$ and $E$. For the normal lung the parameters had more or less an equal influence on the resulting squared error, whereas for the emphysematic lung the parameters $K_{1}$ and $K_{2}$ were far less important for reducing the squared error. 

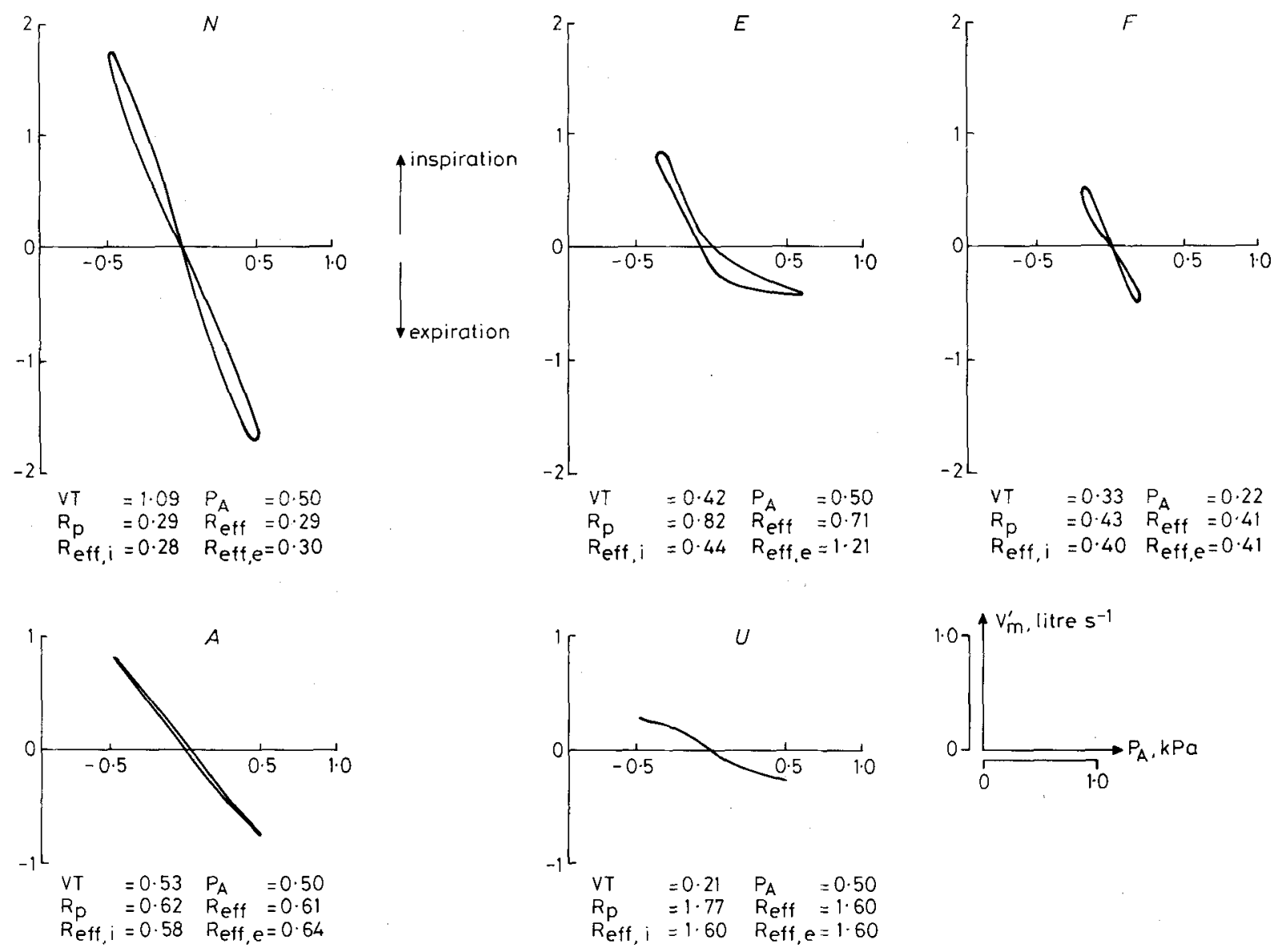

Fig. 4 Simulations performed with model 1 and the parameters of Table 1 for the different groups $N, E, F, A$ and $U$ ( $R$ in $k P a s$ litre $\left.{ }^{-1}\right)$. $V_{T}$ is tidal volume, $P_{A}$ is the amplitude of the simulated pressure curve, $R_{p}$ is the resistance calculated by means of the peak pressure and the associated flow value. $R_{e f f}$ is calculated for the total curve as well as for the inspiratory $\left(R_{e f f, i}\right)$ and expiratory $\left(R_{e f f, e}\right)$ part separately. For symbols see list of symbols and text
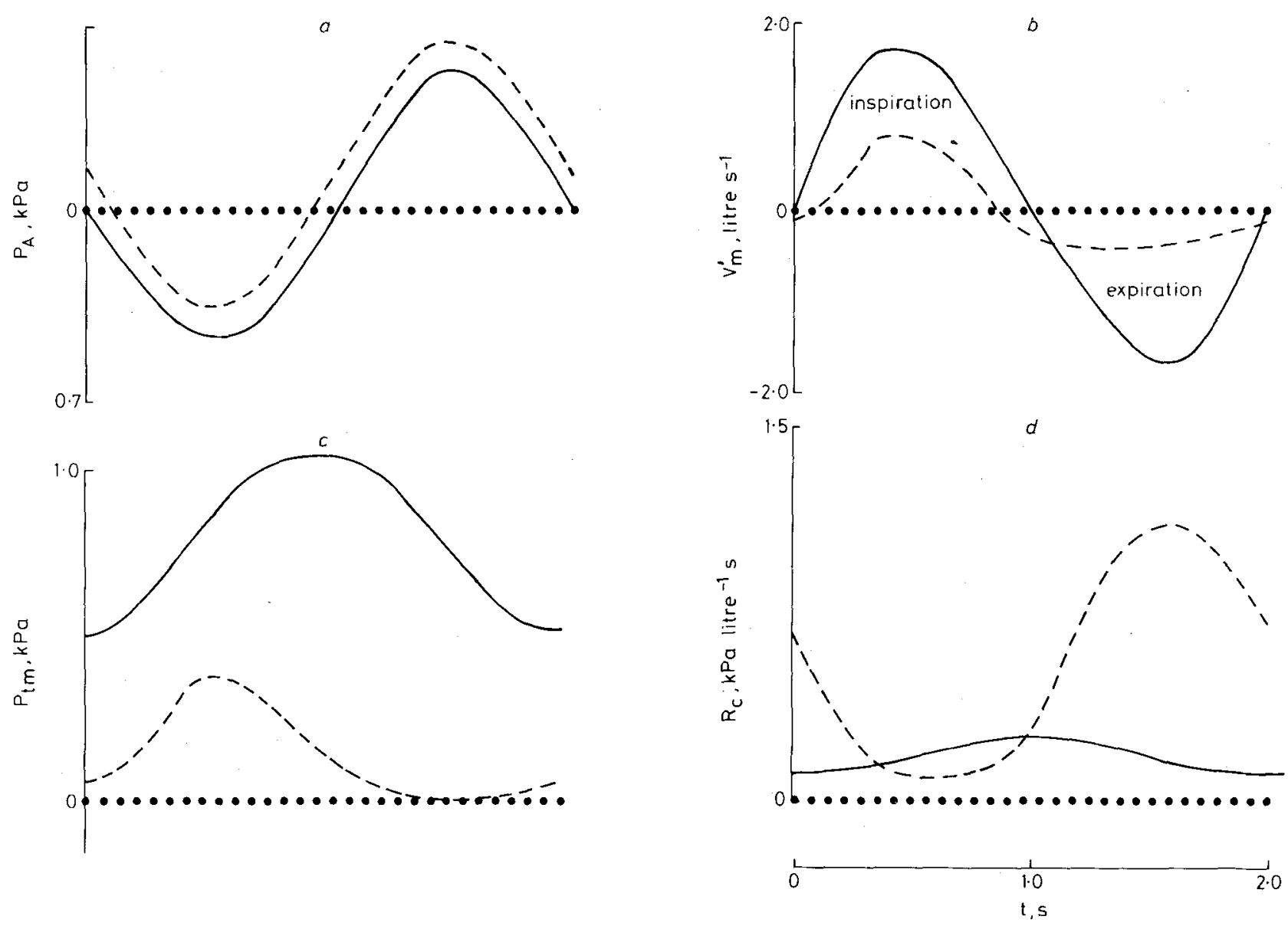

Fig. 5 The variation of different values with time as calculated with model 1 in normal (_) and emphysema (-_-_) during normal breathing. For symbols see list of symbols and text 
Noise superimposed on the model output $V_{m}^{\prime}$ gave no appreciable influence on the parameter estimates. This was not the case when noise was added to the input variable $P_{A}$, as is shown in Table 2 . If the starting values were chosen from the same pathology class or from the normal class the total resistance from the bronchial tree, as given

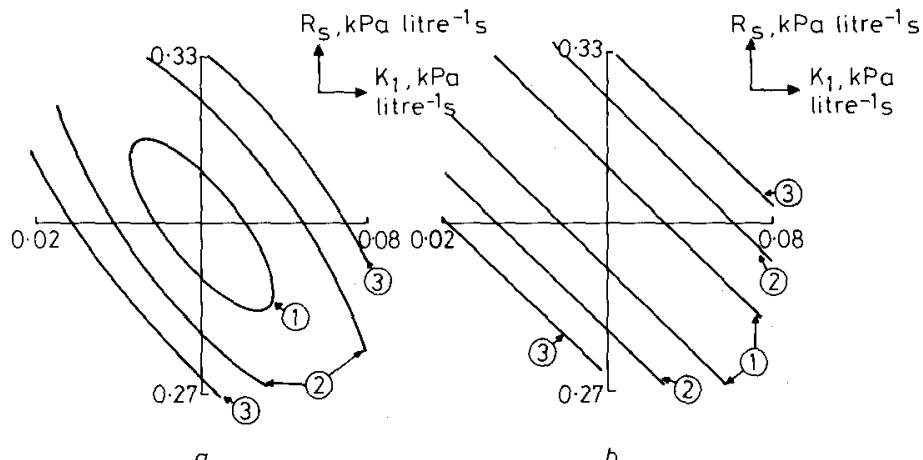

Fig. 6 Plots of contour lines ( $1: E E=0.01 ; 2: E E=0.05,3$ : $E E=0 \cdot 1)$ as function of parameters $K_{1}$ and $R_{s}$ for $a$ highly collapsible $\left(P_{L}=0 \cdot 1\right)$ and a stiff compressible segment $\left(P_{L}=0.9\right)$, respectively. Optimal parameter values: $K_{1}=0.05 ; K_{2}=0.02 ; R_{s}=0.3$ and $P_{L}=0.1$ in (a) and $P_{L}=0.9$ in (b). For these calculations the influence of volume changes on $P_{L}$ was neglected $(1 / C=0)$. In both figures: $x$-axis $0.02<K_{1}<0.08$ ( $\left(\mathrm{Palitre}^{-1} \mathrm{~s}\right)$; $y$-axis $0.27<R_{s}<0.33$ (kPalitre ${ }^{-1}$ s). For symbols see list of symbols and text

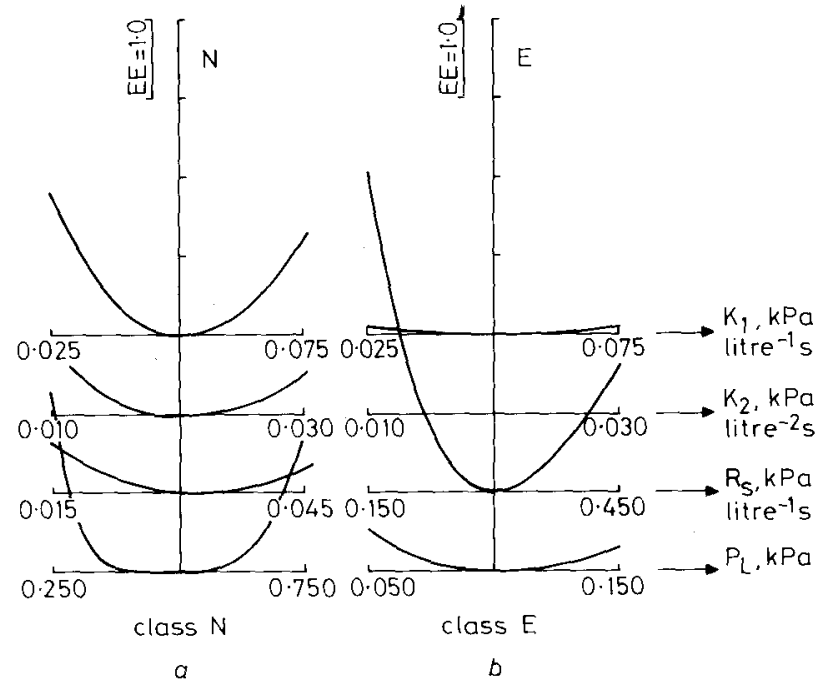

Fig. 7 Plots of EE as function of a perturbation plus and minus 50 per cent of the parameter for the normal and emphysema case. Remaining parameters have the value belonging to the class value. For these calculations the influence of volume change on change in $P_{L}$ was neglected $(1 / C=0)$. (a) Class $N$; (b) class E. For symbols see list of symbols and text by the effective resistance, appeared to be not influenced by random noise. For the $N, E$ and $F$ classes hardly any differences were found in all parameters. The $A$ and $U$ classes showed somewhat larger differences for all parameters. Except for the $A$ and $U$ class, it was possible to obtain an accurate $P_{L}$ estimate in all situations. The same was true for the $N$ class if the $E$-parameters were taken as starting values; although, as mentioned above, $R_{\text {eff }}$ was not influenced by the separation in $R_{\mathrm{s}}$ and upper airway resistance. This last value as characterised by $K_{1}$ and $K_{2}$ was less accurate.

Table 3 shows the parameter estimates for the complex model (1) and those of the three simplified models. The parameters of the simplified models (2, 3 and 4) were obtained by fitting these models to the curves simulated with the complex model (1). This was done for the five separate classes $(N, E, A, F$ and $U)$. In these simulations noise was not superimposed. The estimation with model 1 agrees with the values given in Table 2 in the case of absence of noise. Except for the application of model 2 (one linear resistance) in the emphysema case, in all other simplified model fits the estimate for the effective resistance of the total respiratory cycle was close to the effective resistance calculated with the complex model. Because of assymetries of the inspiratory and expiratory part of the curve in the emphysema class a separate resistance for each part (model 3) was necessary to obtain a reliable estimate of the effective resistance for the whole cycle.

Although most obvious for the $U$ class, in all cases model 4 with a linear and tubulent resistance parameter for inspiration and expiration gave a good fit based on the square error $(E E)$. Only for the class $U$ curve and the expiratory part of the class $E$ curve were the parameters for the turbulent resistance part $\left(K_{7}\right.$ and $\left.K_{9}\right)$ markedly larger then those for the linear part $\left(K_{6}\right.$ and $\left.K_{8}\right)$. In the emphysema case the expiratory curve was largely determined by airway compression. In all other cases the turbulent resistance parameters were much smaller than the linear ones.

\section{Discussion}

Estimation of pulmonary parameters based on model fitting of body plethysmographic pressure/flow tracings has been performed by a small number of investigators (FEINBERG et al., 1970; Golden et al. 1973). These attempts provided a better understanding of body plethysmographic alveolar pressure/flow data. In this present study an extended version of the serial lung model of GoLDEN $e t$ al., (1973) has been used.

The aim of this present study was (1) to evaluate a physiologically well defined serial lung model with respect to its ability to simulate patient curves, and (2) to study the

Table 2 Residual square error $(E E)$, parameter estimates $\left(K_{1}, K_{2}, R_{s}\right.$ and $\left.P_{L}\right)$ and effective resistance estimate ( $\left.R_{\text {eff }}\right)$ with and without addition of random noise of $0.02 \mathrm{kPa}$ to the input signal $P_{A}$ and of 0.02 litre $\mathrm{s}^{-1}$ to $V_{m}^{\prime}$. In the table the class of which the start parameters were taken is indicated. For each pathology class input parameters were as defined in Table $l$ and starting values were chosen both according to the same class and to the normal class. For further explanation see text

\begin{tabular}{|c|c|c|c|c|c|c|c|c|c|c|c|c|c|c|c|}
\hline Simulate class & & $N$ & & & $E$ & & & $F$ & & & $A$ & & & $U$ & \\
\hline Noise level & 0 & 0.2 & 0.2 & 0 & 0.2 & 0.2 & 0 & 0.2 & 0.2 & 0 & 0.2 & $0 \cdot 2$ & 0 & $0 \cdot 2$ & 0.2 \\
\hline Start class & $N$ & $N$ & $E$ & $E$ & $E$ & $N$ & $F$ & $F$ & $N$ & $A$ & $A$ & $N$ & $U$ & $U$ & $N$ \\
\hline$E E$ & 0.00 & 0.33 & 0.33 & 0.00 & 0.27 & $0 \cdot 26$ & 0.00 & $0 \cdot 18$ & $0 \cdot 18$ & 0.00 & $0 \cdot 20$ & $0 \cdot 17$ & 0.00 & 0.03 & 0.03 \\
\hline$K_{1}$ & 0.05 & 0.05 & 0.04 & 0.05 & 0.05 & 0.01 & 0.05 & 0.05 & 0.04 & $0 \cdot 10$ & $0 \cdot 13$ & $0 \cdot 20$ & $0 \cdot 20$ & $0 \cdot 29$ & $0 \cdot 40$ \\
\hline$K_{2}$ & 0.02 & 0.02 & 0.03 & 0.02 & 0.03 & 0.08 & 0.02 & 0.05 & $0 \cdot 05$ & $0 \cdot 06$ & 0.09 & $0 \cdot 10$ & $4 \cdot 6$ & $4 \cdot 6$ & $4 \cdot 14$ \\
\hline$R_{\mathrm{S}}$ & 0.03 & 0.03 & 0.03 & $0 \cdot 30$ & $0 \cdot 30$ & $0 \cdot 30$ & 0.03 & 0.02 & 0.02 & $0 \cdot 30$ & $0 \cdot 26$ & $0 \cdot 19$ & 0.03 & 0.03 & 0.00 \\
\hline$P L$ & 0.50 & 0.50 & 0.50 & $0 \cdot 10$ & $0 \cdot 10$ & $0 \cdot 10$ & 0.90 & 0.90 & 0.90 & 0.50 & 0.40 & $0 \cdot 34$ & 0.50 & 0.50 & 0.55 \\
\hline$R_{e f f}$ & 0.29 & 0.29 & 0.29 & 0.71 & 0.72 & 0.72 & 0.41 & 0.41 & 0.41 & $0 \cdot 61$ & 0.61 & 0.61 & $1 \cdot 60$ & $1 \cdot 60$ & 1.59 \\
\hline
\end{tabular}


Table 3 Results of the estimation process for the primary (I) and the simplified (2,3 and 4) models, performed on signals generated by means of a simulation with mode 1 . Indicated are for each class and each fitted model the resulting square error, the total effective resistance $\left(R_{\text {eff }}\right)$, the effective resistance for inspiration and expiration separately $\left(R_{e f f, i n}\right.$ and $\left.R_{\text {eff,ex }}\right)$ and the resistance line through the extreme points $\left(R_{p}\right)$

\begin{tabular}{|c|c|c|c|c|c|c|c|}
\hline Class & Model & Parameter values & $E E$ & $R_{e f f}$ & $R_{e f f, \text { in }}$ & $R_{e f f, e x}$ & $R_{p}$ \\
\hline \multirow[t]{4}{*}{$N$} & 1 & $K_{1}=0.05 K_{2}=0.02 \quad R_{s}=0.03 P_{1}=0.50$ & 0.00 & 0.29 & $0 \cdot 28$ & $0 \cdot 30$ & $0 \cdot 29$ \\
\hline & 2 & $K_{3}=0.29$ & 3.69 & 0.29 & $0 \cdot 29$ & 0.29 & 0.29 \\
\hline & 3 & $K_{4}=0.29 K_{5}=0.30$ & $3 \cdot 55$ & $0 \cdot 29$ & $0 \cdot 29$ & $0 \cdot 30$ & $0 \cdot 29$ \\
\hline & 4 & $K_{6}=0.29 K_{7}=0.00 K_{8}=0.28 K_{9}=0.01$ & $3 \cdot 54$ & 0.29 & 0.29 & $0 \cdot 30$ & $0 \cdot 30$ \\
\hline \multirow[t]{4}{*}{$E$} & 1 & $K_{1}=0.05 K_{2}=0.02 R_{s}=0.30 P L=0.10$ & 0.00 & $0 \cdot 71$ & $0 \cdot 44$ & $1 \cdot 21$ & 0.82 \\
\hline & 2 & $K_{3}=0.98$ & $11 \cdot 2$ & $0 \cdot 98$ & 0.97 & 0.97 & 0.98 \\
\hline & 3 & $K_{4}=0.45 K_{5}=1.32$ & $2 \cdot 06$ & $0 \cdot 75$ & $0 \cdot 45$ & $1 \cdot 32$ & $0 \cdot 79$ \\
\hline & 4 & $K_{6}=0.45 K_{7}=0.01 K_{8}=0.01 K_{9}=3.29$ & $1 \cdot 52$ & $0 \cdot 74$ & $0 \cdot 45$ & $1 \cdot 27$ & $0 \cdot 81$ \\
\hline \multirow[t]{4}{*}{$F$} & 1 & $K_{1}^{0}=0.05 K_{2}=0.03 \quad R_{s}^{\circ}=0.03 P l=0.90$ & $0 \cdot 00$ & $0 \cdot 41$ & $0 \cdot 40$ & $0 \cdot 41$ & $0 \cdot 43$ \\
\hline & 2 & $K_{3}=0.43$ & $1 \cdot 36$ & $0 \cdot 43$ & $0 \cdot 43$ & $0 \cdot 43$ & $0 \cdot 43$ \\
\hline & 3 & $K_{4}=0.42 K_{5}=0.43$ & $1 \cdot 36$ & $0 \cdot 43$ & $0 \cdot 42$ & $0 \cdot 43$ & $0 \cdot 43$ \\
\hline & 4 & $K_{6}=0.37 K_{7}=0.10 K_{8}=0.43 K_{9}=0.00$ & $1 \cdot 35$ & 0.42 & 0.42 & $0 \cdot 43$ & $0 \cdot 43$ \\
\hline \multirow[t]{4}{*}{$A$} & 1 & $K_{1}=0.10 K_{2}=0.06 \quad R_{s}=0.30 \mathrm{Pl}=0.50$ & 0.00 & $0 \cdot 61$ & $0 \cdot 58$ & 0.64 & 0.62 \\
\hline & 2 & $K_{3}=0.61$ & $0 \cdot 32$ & 0.61 & $0 \cdot 61$ & 0.61 & 0.61 \\
\hline & 3 & $K_{4}=0.58 K_{5}=0.64$ & $0 \cdot 14$ & 0.61 & 0.58 & 0.64 & 0.61 \\
\hline & 4 & $K_{6}=0.53 K_{7}=0.07 K_{8}=0.60 K_{9}=0.06$ & $0 \cdot 12$ & 0.61 & $0 \cdot 58$ & 0.64 & $0 \cdot 61$ \\
\hline \multirow[t]{4}{*}{$U$} & 1 & $K_{1}=0.20 K_{2}=4.60 R_{s}=0.30 P l=0.50$ & 0.00 & 1.60 & 1.60 & $1 \cdot 60$ & $1 \cdot 76$ \\
\hline & 2 & $K_{3}=1.62$ & $0 \cdot 15$ & 1.62 & $1 \cdot 62$ & 1.62 & $1 \cdot 62$ \\
\hline & 3 & $K_{4}=1.62 K_{5}=1.62$ & $0 \cdot 15$ & $1 \cdot 62$ & $1 \cdot 62$ & $1 \cdot 62$ & $1 \cdot 62$ \\
\hline & 4 & $K_{6}=0.51 K_{7}=4.34 R_{8}=0.26 K_{9}=5.43$ & $0 \cdot 00$ & $1 \cdot 59$ & 1.59 & 1.60 & $1 \cdot 77$ \\
\hline
\end{tabular}

uniqueness and sensitivity of a parameter estimation technique for the fitting of the simulated curves.

The behaviour of the serial model was studied by simulating different combinations of parameters (Table 1). The model produced patterns (Fig. 4) for the different types of pathology which agreed quite well with the patterns as defined by MATTHYS (1971). In the classes representing the normal condition and patients with fibrosis, asthma and an increased upper airway resistance, a nonlinear behaviour of the model was virtually absent because the influence of the compressible segment is negligible, since either $R_{s}$ was small or $P_{L}$ was relatively large. For emphysema, where both $R_{s}$ was increased and the $P_{L}$ was decreased the effect of the compressible segment was more pronounced. Large differences in the time course of some output variables were found (Fig. 5) between the classes for the normal condition and for emphysema, respectively:

(i) the transmural pressure in class $E$ was below zero during part of the breathing cycle, which indicates the presence of a marked compression even during the normal breathing

(ii) the area of the compressible segment in class $E$ decreased greatly, and, therefore, resistance $R_{c}$ was much larger during expiration than it was during expiration in the normal case (Fig. 4e).

Also the difference in the values of effective resistance, calculated for the inspiratory $\left(R_{e f f, \text { in }}\right)$ and expiratory $\left(R_{e f f, e x}\right)$ resistance, showed this effect of large expiratory airway compression. The model appeared to be sufficiently accurate for simulation of the characteristic loop patterns, related to pulmonary pathology, in spite of a (for practical purposes necessary) simplified description of the compressible segment.

For simulations without additional noise the parameter estimation routine gave unique solutions for the parameter values belonging to each class. It was shown that the sensitivity of the parameter estimation technique depended on the values of the parameters of each class (Fig. 7). In the normal condition (class $N$ ) the sensitivity for the different parameters was more or less the same. For emphysema (class $E$ ) the parameters $R_{s}$ and $P_{L}$ were much more important than $K_{1}$ and $K_{2}$. Thus $K_{1}$ and $K_{2}$ appeared to be of little importance in reducing the error in the parameter estimation scheme. Therefore, the accuracy of the estimation of $K_{1}$ and $K_{2}$ will be smaller than that of both other parameters (Table 2, Figs. $6 a$ and $6 b$ ). The application of noise confirmed the results on the sensitivity of the model fit for the various parameters in dependence on the pathology class. In the case of upper airway obstruction the factor $K_{2}$ has such a dominant influence that the other parameters have almost no influence on the resulting effective resistance and on the shape of the curve.

In circumstances where the influence of the compressible segment was minimal, the serial long model could be simplified. In such situations, a good estimation of the different parameter values in model 1 , which characterised the resistance of the serial connection of the segments, was not possible. Then a model with only one serial resistance element with a laminar and a turbulent term could be used, as was shown in Table 3. This did not hold for the $E$ case where the four models yield appreciably different estimates.

We conclude that the serial lung model is able to fit curves which are influenced by a serial mechanical inhomogeneity, especially defined by the behaviour of the compressible segment. Moreover, parameter estimation was able to recognise in the cases described, even in the presence of noise on the model input, the most relevant aspects of the lung mechanical behaviour.

Acknowledgment - This study was supported by a grant from the Dutch Asthma Foundation.

\section{References}

BanerJee, M., Evans, J. N. and JAEger, M. J. (1976) Uneven ventilation in smokers. Respirat. Physiol., 27, 277-291.

BEKEY, G. A. and BENEKEN, J. E. W. (1978) Identification of biological systems: a survey. Automatica, 14, 41-47.

Bogaard, J. M., Pauw, K. H., Versprille, A., Stam, H., VerBRAAK, A. F. M. and MAAS, A. J. J. (1987a) Maximal expiratory and inspiratory flow-volume curves in bilateral vocal-cord paralysis. $O R L, 49,35-41$.

Bogaard, J. M., Pauw, K. H. and Versprille, A. (1987b) Flow 
limitation in upper airway obstruction (theoretical analysis) Ibid., 49, 42-47.

Cettl, L., Dvorak, J., Felkel, H. and Feuereisl, R. (1979) Results of simulation of nonhomogeneous ventilatory mechanics for a patient-computer arrangement. Int. J. Bio-Med. Comput., 10, 67-74.

COTES, J. E. (1975) Lung function. Assessment and application in medicine. Blackwell Scientific Publications.

Dubois, A. B., Botelho, S. Y. and Comroe, J. H. Jr. (1956) A new method for measuring airway resistance in man using a bodyplethysmograph values in normal subjects and in patients with respiratory disease. J. Clin. Invest., 35, 327-335.

Feinberg, B. N., Chester, E. H. and Schoeffler, J. D. (1970) Parameter estimation: a diagnostic aid for lung diseases. Instrum. Technol., 40-46.

Feinberg, B. N. and Chester, E. H. (1972) A dynamic model of pulmonary mechanics to simulate a panting maneuver. Bull. Physio-path. Respirat., 8, 305-322.

Golden, J. F., Clark, J. W. Jr., and Stevens, P. M. (1973) Mathematical modeling of pulmonary airway dynamics. IEEE Trans., BME-20, 397-404.

Guyatt, A. H., Alpers, J. H., Hill, I. D. and Bramley, A. C. (1967) Variability of plethysmographic measurements of airway resistance in man. J. Appl. Physiol, 22, 383-389.

Holland, W. P. J., Verbraak, A. F. M., Bogaard, J. M. and BOENDER, W. (1986) Effective airway resistance: a reliable variable from bodyplethysmography (A theoretical analysis and an application in COLD patients). Clin. Physics \& Physiol. Meas., 7, 319-331.

Hyatt, R. E. and Flath, R. E. (1966) Influence of lung parenchyma on pressure-diameter behaviour of dog bronchi. $J$. Appl. Physiol., 21, 1448-1452.

HyatT, R. E. (1983) Expiratory flow limitation. J. Appl. Physiol.: Respirat. Environ. Exercise Physiol., 55, 1-8.

Lambert, R. K., Wilson, T. A., Hyatt, R. E. and Rodarte, J. R. (1982) A computational model for expiratory flow. Ibid, 52 44-56.

MACKLEM, P. T. and MEAD, J. (1968) Factors determining maximum expiratory flow in dogs. J. Appl. Physiol., 25, 159 169.

MARQUARDT, D. W. (1963) An algorithm for least-squares estimation of nonlinear parameters. SIAM J., 11, 431-441.

Martin, H. B. and Proctor, D. F. (1958) Pressure-volume measurements on dog bronchi. J. Appl. Physiol., 13, 337-343.

Martin, C. J., YounG, A. C. and Ishikawa, K. (1965) Regional lung mechanics in pulmonary disease. J. Clin. Invest., 44, $906-$ 913.

Matthys, H. (1971) Ganzkörperplethysmographie. Pneumonology $1,146,216-231$.

MATTHYS, H., FisCher, J., UlRiChS, H.Ch. and RüHLE, K. H. (1979) Functional patterns of different lung diseases for computer-assisted diagnostic procedures. Progr. Respirat. Res., 11, 188-201.

Mead, J., Turner, J. M., Macklem, P. T. and Little, J. B. (1967) Significance of the relationship between lung recoil and maximum expiratory flow. J. Appl. Physiol., 22, 95-108.

Murtagh, P. S., Proctor, D. F., Permutt, S., Kelly, B. L. and EVERING, S. (1971) Bronchial mechanics in excised dog lobes. Ibid., 31, 403-408.

Otis, A. B., McKerrow, C. B., Bartlett, R. A., Mead, J. McIlroy, M. B., Selverstone, N. J. and Radford, E. P. Jr (1956) Mechanical factors in distribution of pulmonary ventilation. J. Appl. Physiol., 8, 427-443.

Pride, N. B., Permutt, S., Riley, R. L. and BrombergerBARNEA, B. (1967) Determinants of maximal expiratory flow from the lungs. Ibid., 23, 646-662.

PRIDE, N. B. (1971) The assessment of airflow obstruction (role of measurements of airway resistance and of tests of forced expirations). Br. J. Dis. Chest., 65, 135-169.

Reiner T, M., Heise, D. and Trendelenburg, F. (1975) Zum Auswertemodus phasenverschobener Resistancekurven. Pneumol ogie, 152, 147-156

ROHRER, F. (1915) Der Strömungswiderstand in der menschlichen Atemwegen und der Einfluss der unregelmässigen Verzweigung des Bronchialsystems auf der Atmungsverlauf in verschieden Lungenbezirken. Pflügers. Arch. Ges. Physiol., 162, 225-259.
Smidt, U., Finkenzeller, P. and Rennings, C. (1975) On-line Computereinsatz in der Ganzkörperplethysmographie zur Berechnung der mittleren Resistance. Pneumologie, 151, 223 231.

Ulmer, W. T. and ReIF, E. (1965) Die obstruktiven Erkrankungen der Atemwege. Klinische Bedeutung und objektiver Nachweis mit der Ganzkörperplethysmographie. Dtsch. Med. Wschr., 90, Jg. 41.

\section{Authors' biographies}

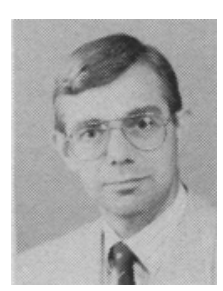

Ton F. M. Verbraak was born in Tilburg, The Netherlands in 1946. He received a MEE degree from Eindhoven University of Technology, Division of Medical Electrical Engineering, in 1976. He works in the Pulmonary Function Department of the University Hospital Rotterdam. The present paper is part of a thesis on signal processing in pulmonary function and simulation and parameter estimation in body plethysmography. His fields of interest are signal processing, physiological modelling and patient monitoring.

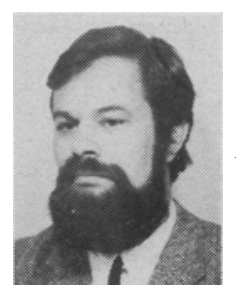

Jan M. Bogaard was born in The Hague in 1936. He worked for 15 years as chemical engineer (Ing.) in the Department of Lung Function \& Exercise Testing at the University of Leiden. During the same period he became an experimental physicist at this university. From 1974 he worked in Rotterdam where he received his $\mathrm{Ph} . \mathrm{D}$. degree in 1980 for work on random walk modelling of indicator-dilution curves. His fields of interests are development and application of methods on pulmonary function, regulation of breathing and exercise physiology.

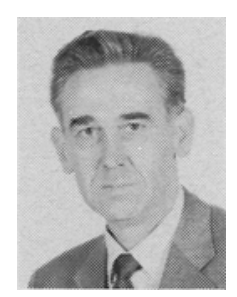

Jan E. W. Beneken received a MEE degree from Delft University of Technology in 1958 and a Ph.D. degree in Experimental Physics from the University of Utrecht. He is presently Professor and Chairman of the Division of Medical Electrical Engineering of Eindhoven University of Technology with research interests in physiological modelling, patient monitoring. AI applications in medicine, ultrasound transmission tomography and communication aids for the handicapped. $\mathrm{He}$ is Chairman of the Bioengineering Committee (COMAC-BME) of the European Community.

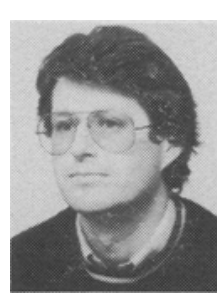

Ed Hoorn was born in Rotterdam, The Netherlands in 1955. He received his Ing. degree in Physical Engineering. In 1980 he worked at the Pathophysiological Laboratory of the Erasmus University. Currently he works at the Central Department of Automation \& Information of the University Hospital Rotterdam, where he is involved in the development of computer programs for clinical routine and research. His fields of interest are computerised data acquisition and data analysis.

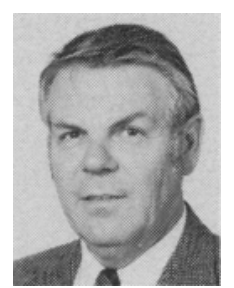

Professor Adrian Versprille is a full professor in clinical respiratory physiology and head of the research laboratory of the Department of Pulmonary Diseases, Erasmus University, Rotterdam and head of the pulmonary laboratory University Hospital 'Dijkzigt'. The field of mechanical ventilation and the development of diagnostic and therapeutic methods as a spinoff of his basic research are his main interests. 